\title{
Effect of an external magnetic field on the determination of E1M1 two-photon decay rates in Be-like ions
}

\author{
Jon Grumer, ${ }^{1, *}$ Wenxian Li ${ }^{2}$ Dietrich Bernhardt, ${ }^{3}$ Jiguang Li,,${ }^{1}$ Stefan Schippers, ${ }^{3}$ Tomas Brage,,${ }^{1}$ \\ Per Jönsson, ${ }^{4}$ Roger Hutton, ${ }^{2}$ and Yaming Zou ${ }^{2}$ \\ ${ }^{1}$ Division of Mathematical Physics, Department of Physics, Lund University, S-221 00 Lund, Sweden \\ ${ }^{2}$ Institute of Modern Physics, Fudan University, 200433 Shanghai, China \\ ${ }^{3}$ Institut für Atom- und Molekülphysik, Justus-Liebig-Universität Giessen, 35392 Giessen, Germany \\ ${ }^{4}$ Group for Materials Science and Applied Mathematics, Malmö University, S-205 06 Malmö, Sweden
}

(Received 11 July 2013; published 22 August 2013)

\begin{abstract}
In this work we report on ab initio theoretical results for the magnetic-field-induced $2 s 2 p^{3} P_{0} \rightarrow 2 s^{21} S_{0}$ E1 transition for ions in the beryllium isoelectronic sequence between $Z=5$ and 92 . It has been proposed that the rate of the E1M1 two-photon transition $2 s 2 p^{3} P_{0} \rightarrow 2 s^{21} S_{0}$ can be extracted from the lifetime of the ${ }^{3} P_{0}$ state in Be-like ions with zero nuclear spin by employing resonant recombination in a storage ring. This experimental approach involves a perturbing external magnetic field. The effect of this field needs to be evaluated in order to properly extract the two-photon rate from the measured decay curves. The magnetic-field-induced transition rates are carefully evaluated, and it is shown that, with a typical storage-ring field strength, it is dominant or of the same order as the E1M1 rate for low- and mid- $Z$ ions. Results for several field strengths and ions are presented, and we also give a simple $Z$-dependent formula for the rate. We estimate the uncertainties of our model to be within $5 \%$ for low- and mid- $Z$ ions and slightly larger for more highly charged ions. Furthermore we evaluate the importance of including both perturber states, ${ }^{3} P_{1}$ and ${ }^{1} P_{1}$, and it is shown that excluding the influence of the ${ }^{1} P_{1}$ perturber overestimates the rate by up to $26 \%$ for the mid- $Z$ ions.
\end{abstract}

DOI: 10.1103/PhysRevA.88.022513

PACS number(s): 31.15.ag, 32.60.+i

\section{INTRODUCTION}

Two-photon transitions are exotic decay modes in atoms and ions. Nevertheless, they are of practical interest, e.g., in astrophysics where the $2 s \rightarrow 1 s$ (2E1) transition in hydrogen contributes to the observed continuum radiation from planetary nebulae [1], Herbig-Haro objects [2], and H II regions [3]. Theoretical work on two-photon transitions started at the dawn of quantum mechanics [4]. Since then, theoretical and experimental work has mainly focused on $\mathrm{H}$-like and He-like systems ([5], and references therein). Various aspects of two-photon transitions, such as resonance effects [6], negative continuum effects [7], relativistic and QED effects [8], and higher-order multipole effects [9] on two-photon transitions in $\mathrm{H}$-like ions, in these isoelectronic sequences of ions have been addressed in very recent (mostly theoretical) studies. Additionally, the sensitivity of the spectral shape of the emitted photon continuum to relativistic effects [10] and angular correlations [11] and quantum correlations [12] between the two-photons have been investigated.

In He-like ions there exist three long-lived metastable states which decay (partly) via the two-photon transitions $1 s 2 s^{1} S_{0} \rightarrow 1 s^{2} S_{0}$ (2E1), $1 s 2 s^{3} S_{1} \rightarrow 1 s^{2} S_{0}$ (2E1), and $1 s 2 p^{3} P_{0} \rightarrow 1 s^{21} S_{0}$ (E1M1). The latter competes with the dominating $1 s 2 p^{3} P_{0} \rightarrow 1 s 2 s^{3} S_{1}$ one-photon E1 transition. The relative importance of the E1M1 transition increases with nuclear charge $Z$. For $Z=92$ the E1M1 branching ratio has been calculated to amount to about $32 \%[13,14]$.

With Be-like ions the situation is much more clear-cut. The $2 s 2 p^{3} P_{0}$ state is the lowest excited state, and for isotopes with a nonzero nuclear spin, the $J=0 \rightarrow 0$ transition channel

\footnotetext{
*jon.grumer@teorfys.lu.se
}

opens up due to mixing of the hyperfine levels, leading to a so-called hyperfine-induced transition (HIT). Such a shortening of lifetimes of metastable states owing to hyperfine interaction is referred to as hyperfine quenching and has been investigated for Be-like ions both theoretically [15-20] and experimentally $[21,22]$. For isotopes with zero nuclear spin, on the other hand, a one-photon transition to the ground state $2 s^{2} S_{0}$ is strictly forbidden in a field-free region, and the lowest-order decay channel is a very slow E1M1 two-photon process. The most important third-order process is a 3E1 three-photon decay, which has a transition rate smaller than the two-photon process by a factor of $\alpha$, the fine-structure constant, according to Laughlin [23].

The calculation of E1M1 rates involves potentially significant negative-energy contributions to the transition amplitudes [13]. Thus, an accurate measurement of the experimental decay rate would constitute an ultimate benchmark of relativistic many-body theoretical methods and computational schemes. So far no experimental observations of E1M1 transitions in He-like or Be-like systems exist [5].

Recently, future storage-ring experiments have been proposed [24-26] to measure $2 s 2 p^{3} P_{0} \rightarrow 2 s^{2}{ }^{1} S_{0}$ E1M1 twophoton transition rates for heavy Be-like ions with nuclear charges $Z \gtrsim 50$. However, the magnetic field of the storagering dipole magnets will give rise to a magnetic-field-induced E1 transition (from here on referred to as a MIT), possibly with a rate of the same order of magnitude as the rate of the two-photon transition. Hence, to correctly deduce the two-photon rate from such an experiment, there is a need for accurate MIT rates as discussed in Ref. [26].

Arguably, a MIT was observed for the first time in 2003 by Beiersdorfer et al. in Ne-like Ar, using the EBIT-II Electron Beam Ion Trap at the Lawrence Livermore National 
Laboratory [27]. They also showed that such transitions can play an important role in high-temperature plasma diagnostics, e.g., in fusion reactors.

In the present work we investigate the mixing, as induced by an external magnetic field, of the four atomic fine-structure states $2 s 2 p^{1,3} P_{0,1,2}$ in Be-like ions with zero nuclear spin, giving rise to a MIT from ${ }^{3} P_{0}$ to the ground state. Note that there will also be a MIT from ${ }^{3} P_{2}$ to the ground state, which is of no direct interest to this work.

In the following section we introduce the relevant details of storage-ring measurements of atomic lifetimes. Our theoretical methods are described in Sec. III, and computational details are given in Sec. IV. Our results and conclusions are presented in Secs. V and VI.

\section{STORAGE-RING MEASUREMENTS OF ATOMIC LIFETIMES}

Heavy-ion storage rings are ideal devices for measuring atomic lifetimes [28]. They provide a unique experimental environment which is characterized by low residual gas density and correspondingly long ion storage times of up to several hours [29]. In a typical experiment, ions with well-defined charge state, mass, and kinetic energy are injected into the storage ring from an external accelerator. Beam-cooling techniques such as electron cooling [30] or stochastic cooling [31] may be applied to reduce internal energy spread and the diameter of the stored ion beam, i.e., to create wellcontrolled experimental conditions. Long-lived metastable levels of interest are usually generated by the charge-stripping process that is used for producing the desired ion charge state in the accelerator. Metastable levels may also be populated in situ by collisional excitation [32] or by optical pumping [33].

The standard technique for measuring atomic decay rates in an ion storage ring is to monitor the fluorescence from the longlived excited levels as a function of storage time [28]. However, this approach suffers from small solid angles and background photons, which severely hampers the investigation of weak decay channels [34]. An alternative approach is electron-ion collision spectroscopy, where level-specific charge-changing electron-ion collision processes such as dielectronic recombination (DR) are exploited for monitoring the decay of the metastable ion beam fraction [35]. This techniques yields comparatively high signal rates since the fast-moving product ions are confined into a narrow cone and can thus be detected with high efficiency. It has been successfully employed for the measurement of $2 s 2 p^{3} P_{0} \rightarrow 2 s^{2}{ }^{1} S_{0}$ HIT rates in Be-like ${ }^{47} \mathrm{Ti}^{18+}[21]$ and ${ }^{33} \mathrm{~S}^{12+}$ [22] at the Heidelberg heavy-ion Test Storage-Ring (TSR). A proposal has been made [24-26] to use the same technique at the heavy-ion Experimental Storage Ring (ESR), of the GSI Helmholtz Center for Heavy-Ion Research in Darmstadt, Germany, for the measurement of the $2 s 2 p^{3} P_{0} \rightarrow 2 s^{2}{ }^{1} S_{0}$ E1M1 two-photon transition rates in heavy Be-like ions with zero nuclear spin (Fig. 1).

An issue of concern in these measurements is in how far the atomic lifetimes are influenced by the magnetic fields that are generated by the storage-ring dipole, quadrupole, and higher-order correction magnets. Magnetic quenching in the TSR has been investigated theoretically by Li et al. [20] for the $2 s 2 p^{3} P_{0}$ level in Be-like ${ }^{47} \mathrm{Ti}^{18+}$. The effect has been

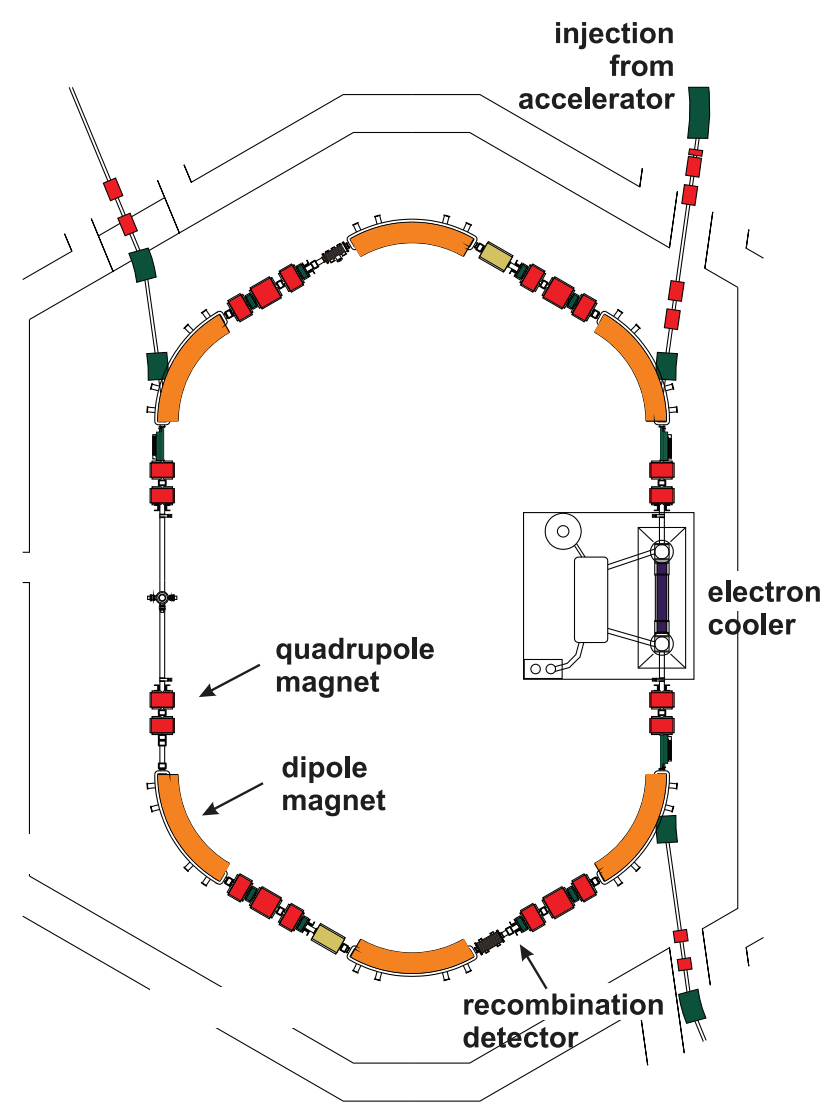

FIG. 1. (Color online) Sketch of the heavy-ion storage-ring ESR of the GSI Helmholtz Center for Heavy-Ion Research in Darmstadt, Germany. The ESR storage ring [36] has a circumference of $108.36 \mathrm{~m}$. It has a hexagonal layout consisting of six dipole bending magnets, two long straight sections, and four short straight sections. The lengths of the long and short straight sections are 18.235 and $8.155 \mathrm{~m}$, respectively. The dipole magnets have a bending angle of $60^{\circ}$ and a bending radius $\rho=6.25 \mathrm{~m}$. The length of the flight path through all dipole magnets is $2 \pi \rho=39.27 \mathrm{~m}$; i.e., the six magnets cover $36.24 \%$ of the ring circumference. The maximum magnetic field $B$ is about $1.5 \mathrm{~T}$.

found to be insignificant for this specific case. The effect of external fields on the HIT rates has also been investigated experimentally. In the $S^{12+}$ storage-ring experiment [22] the magnetic-field strength of the storage-ring dipoles was varied by a factor of 2 . Within the experimental uncertainties no influence of this $B$-field variation on the measured HIT rate was found. In contrast to the HIT and E1M1 transition rates, which, in general, are increasing with $Z$, the MIT rate decreases with $Z$. Hence it can be expected that the relative importance of magnetic quenching decreases with increasing $Z$.

Although the ions, which move at typically $10 \%-30 \%$ of the speed of light $c$, are subjected to an alternating magnetic field with frequencies in the megahertz range, we here as a first approach treat the magnetic field as constant. This implies that we also neglect the magnetic fields of the quadrupole and higher-order correction magnets, which are much weaker than the field in the bending dipole magnets anyway.

It should also be noted that the magnetic field transforms into an electric field $\mathbf{E}=q(\mathbf{v} \times \mathbf{B})$ in the rest frame of the ions, with charge $q$ moving with velocity $\boldsymbol{v}$. Under rather extreme 
experimental conditions, i.e., for $v=c$ and $B=1.5 \mathrm{~T}$, the electric field strength amounts to $4.5 \times 10^{8} \mathrm{~V} \mathrm{~m}^{-1}$. Searching for parity-violating effects, Maul et al. [37] have calculated the rate for the associated quenching of the $2 s 2 p{ }^{3} P_{0}$ level. This rate scales quadratically with field strength $E$. Even for $E=4.5 \times 10^{8} \mathrm{~V} \mathrm{~m}^{-1}$ the effect is very weak. The associated transition rates are smaller than $\sim 2 \times 10^{-5} \mathrm{~s}^{-1}$ [26] and thus are insignificant for the present study.

\section{THEORETICAL METHOD}

The details of the theoretical approach used in this work have been outlined in a recent paper on MITs in Ne-like ions [38]. Here we just briefly summarize the method.

The Hamiltonian of an atom with zero nuclear spin under the influence of an external homogeneous magnetic field $\mathbf{B}$ can be written in the following form [39]:

$$
H=H_{f s}+H_{m}=H_{f s}+\left(\mathbf{N}^{(1)}+\Delta \mathbf{N}^{(1)}\right) \cdot \mathbf{B},
$$

where the first term, $H_{f s}$, is the relativistic fine-structure Hamiltonian which in our approach includes the Breit interaction and leading QED effects, such as self-energy and vacuum polarization. The tensor operator $\mathbf{N}^{(1)}$ represents the coupling of the electrons with the field, and $\Delta \mathbf{N}^{(1)}$ is the Schwinger QED correction. Explicit forms of the operators can be found in Ref. [39].

In the presence of an external magnetic field, $M$ (and parity, which we leave out for simplicity) is the only good electronic quantum number, and we expand the $M$-dependent atomic state functions $|M\rangle$ in terms of field-independent atomic state functions (ASFs), $|\Gamma J M\rangle$, that are eigenstates of the finestructure Hamiltonian:

$$
|M\rangle=\sum_{\Gamma J} d_{\Gamma J}|\Gamma J M\rangle .
$$

The mixing coefficients associated with the magnetic-field perturbation $d_{\Gamma J}$ can be obtained through first-order perturbation theory,

$$
d_{\Gamma J}=\frac{\left\langle\Gamma J M\left|H_{m}\right| \Gamma_{0} J_{0} M_{0}\right\rangle}{E\left(\Gamma_{0} J_{0}\right)-E(\Gamma J)},
$$

where the labels having a subscript zero denote the reference state. Alternatively, one can evaluate the mixing by solving the corresponding eigenvalue problem.

In order to construct the ASFs we use the multiconfiguration Dirac-Hartree-Fock (MCDHF) approach [40]. The starting point of this method is to write the ASFs as linear combinations of configuration state functions (CSFs), which in turn are eigenfunctions of $J^{2}, J_{z}$, and parity,

$$
|\Gamma J M\rangle=\sum_{i} c_{i}\left|\gamma_{i} J M\right\rangle,
$$

where $c_{i}$ are mixing coefficients of the CSFs and $\gamma_{i}$ are labels, such as orbital occupation numbers and intermediate spin-angular couplings, to uniquely define the individual basis functions. Each of these many-electron CSFs are in turn constructed as a coupled antisymmetric sum of products of one-electron wave functions, the Dirac orbitals.

Applying the basis expansion (2), the electric dipole transition probability for a magnetic-field-induced transition

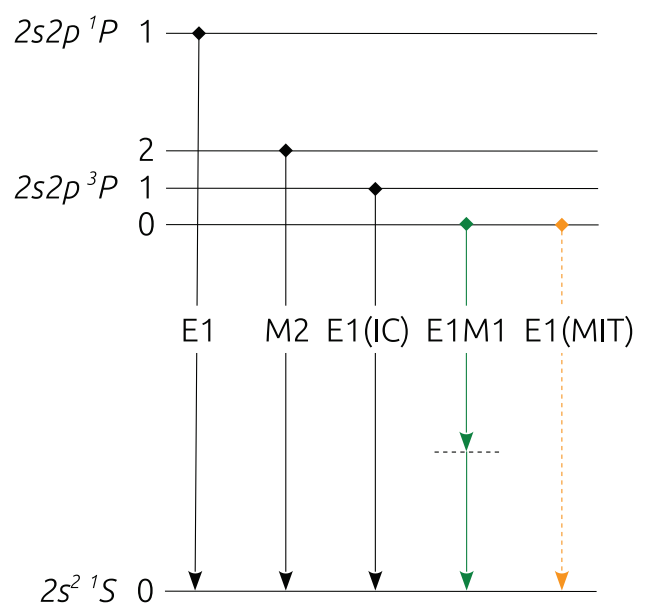

FIG. 2. (Color online) Schematic Grotrian diagram at low $Z$, where $L S$-coupling notation is appropriate, for the lowest states of Be-like ions with zero nuclear spin. The lowest-order decay from ${ }^{3} P_{0}$ is the E1M1 two-photon transition to the ground state. In the presence of an external magnetic field, the usually strictly forbidden one-photon transition channel $2 s 2 p^{3} P_{0} \rightarrow 2 s^{2}{ }^{1} S_{0}$ is opened due to mixing with ${ }^{3} P_{1}, M=0$, which is decaying to the ground state through the unexpected E1 intercombination channel (IC), and with ${ }^{1} P_{1}, M=$ 0 , which decays to the ground state with an allowed E1 transition.

from an initial state $\left|M^{\prime}\right\rangle$ to a final state $|M\rangle$ is given by

$$
\begin{aligned}
A_{\mathrm{MIT}}= & \frac{2.02613 \times 10^{18}}{\lambda^{3}} \sum_{q} \mid \sum_{\Gamma J} \sum_{\Gamma^{\prime} J^{\prime}}(-1)^{J-M} d_{\Gamma J} d_{\Gamma^{\prime} J^{\prime}}^{\prime} \\
& \times\left.\left(\begin{array}{ccc}
J & 1 & J^{\prime} \\
-M & q & M^{\prime}
\end{array}\right)\left\langle\Gamma J\left\|\mathbf{P}^{(1)}\right\| \Gamma^{\prime} J^{\prime}\right\rangle\right|^{2},
\end{aligned}
$$

where $A_{\mathrm{MIT}}$ is in $\mathrm{s}^{-1}$ and $\lambda$ is the wavelength of the transition in $\AA$. One should keep in mind that the real photon energy, that is, the transition energy of the induced transition under consideration (i.e., between $2 s^{2}{ }^{1} S_{0}$ and $2 s 2 p^{3} P_{0}$ in this case), must be used to calculate the electric dipole transition matrix elements [17].

The magnetic interaction induces mixing between states that differ in $J$ by at most 1 ; hence the regular E1 selection rule of change in total angular momentum is extended to $\Delta J=$ $J-J^{\prime}=0, \pm 1, \pm 2, \pm 3$. The mixing also implies that what appears as a $J=0 \rightarrow 0$ transition is allowed.

The general theory can be applied to the MIT rates in Be-like ions. The reference state $2 s 2 p^{3} P_{0}$ in these systems, under the influence of an external magnetic field (see Fig. 2 for schematics of energy structure and possible transition channels), can approximately be expressed as

$$
\begin{aligned}
& \left|" 2 s 2 p^{3} P_{0} " M=0\right\rangle=d_{0}\left|2 s 2 p^{3} P_{0} M=0\right\rangle \\
& +\sum_{\mathcal{S}(=1,3)} d_{\mathcal{S} ; J=1}\left|2 s 2 p^{\mathcal{S}} P_{1} M=0\right\rangle \text {, }
\end{aligned}
$$

where further interactions have been excluded due to large energy separations and relatively weak magnetic interaction couplings. The quotation marks are used to clarify that the notation is just a label corresponding to the largest $J$-dependent 
eigenvector component. The ground state is more or less isolated from other states, so the corresponding $M$-dependent state is very well described by a single ASF,

$$
\text { |" } \left.2 s^{2}{ }^{1} S_{0} " M=0\right\rangle=\left|2 s^{2}{ }^{1} S_{0} M=0\right\rangle .
$$

The inclusion of the perturbing states $\left|2 s 2 p^{1,3} P_{1} M=0\right\rangle$ in the wave function, Eq. (6), opens up one-photon E1 transitions to the ground state. Using Eq. (5) and evaluating the $3 j$ symbol, the corresponding transition rates can be expressed as

$$
\begin{aligned}
A_{\mathrm{MIT}}= & \frac{2.02613 \times 10^{18}}{3 \lambda^{3}} \\
& \times\left|\sum_{\mathcal{S}(=1,3)} d_{\mathcal{S}}\left\langle 2 s 2 p^{1} S_{0} \| \mathbf{P}^{(1)}|| 2 s 2 p^{S} P_{1}\right\rangle\right|^{2} .
\end{aligned}
$$

Finally, since the mixing coefficients $d_{\mathcal{S}}$ in first-order perturbation theory are directly proportional to the magneticfield strength, we define a reduced mixing coefficient $d_{\mathcal{S}}^{R}$ and hence also a reduced transition rate $A_{\mathrm{MIT}}^{R}$, which in effect are independent of $B$, through

$$
d_{\mathcal{S}}=B d_{\mathcal{S}}^{R}, \quad A_{\mathrm{MIT}}=B^{2} A_{\mathrm{MIT}}^{R} .
$$

\section{COMPUTATIONAL MODEL}

\section{A. Summary}

The wave functions of all Be-like ions ranging from boron $(Z=5)$ to uranium $(Z=92)$ are calculated using the latest version of the GRASP2K program suite [41] based on the MCDHF method briefly outlined above.

The radial parts of the Dirac orbitals, together with the expansion coefficients $c_{i}$ in Eq. (4), are optimized in a relativistic self-consistent field (RSCF) procedure based on the Dirac-Coulomb Hamiltonian. This part of the calculation is performed in a layer-by-layer scheme in which the active set of one-electron Dirac orbitals is expanded systematically until satisfactory convergence of atomic properties, such as excitation energies, is achieved.

With a well-optimized basis at hand, the Breit interaction (in the low-frequency limit) and leading QED effects are included in a subsequent relativistic configuration interaction (RCI) model. Both these effects grow in importance with increasing ionization stages compared to the electron correlation, which becomes less important for high- $Z$ ions.

Finally, in order to calculate the transition rate according to Eq. (8), the mixing coefficients $d_{\mathcal{S}}$ have to be evaluated. This is done using the first-order perturbation theory approximation of Eq. (3), with reduced matrix elements calculated using the GRASP2K module HFSZEEMAN [42].

\section{B. Optimization of Dirac orbitals and electron correlation model}

The ASFs of the even-parity, $2 s^{2}{ }^{1} S_{0}$, and the odd-parity states, $2 s 2 p^{3} P_{0,1,2}, 2 s 2 p^{1} P_{1}$, are determined in two separate calculations. The four odd ASFs are determined simultaneously in an extended optimal level (EOL) scheme [43], where the optimization is on a weighted sum of the corresponding fine-structure energies. It should be noted that standard Racah algebra assumes the ASFs to be built from the same set of orthogonal radial orbitals. Thus to compute transition matrix elements between the even- and odd-parity ASFs, generated from independently optimized orbital sets, we apply biorthogonal transformation techniques $[44,45]$, after which the calculation can be performed using standard methods.

We use a correlation model in which the CSF space is generated using a complete active space (CAS) approach with orbitals up to $n=4$ and is then merged with the result of single (S) and double (D) substitutions to higher $n$ 's (with orbital angular momentum restricted by $l \leqslant 6$ ) from the multireference (MR) $\left\{2 s^{2}, 2 p^{2}\right\}$ for the even-parity states and the $\{2 s 2 p\}$ reference for the odd states.

In order to capture as much correlation as possible in the computationally much less demanding RCI calculation, we extend the active space model from above by allowing also for triple $(\mathrm{T})$ and quadruple $(\mathrm{Q})$ substitutions with the restriction, in excess of the orbital angular momentum upper limit, that there should always be at least two electrons in subshells with $n \leqslant 3$. This is in effect a simple way of generating a SD expansion from a large MR.

The active space for ions with charge states $Z=5$ to 42 is expanded up to $n=8$ according to the rules set up above, corresponding to a maximum (in the RCI calculation) of 37653 and 296215 CSFs of even $(J=0)$ and odd parity $(J=0,1,2)$, respectively. These calculations include 62 Dirac orbitals. For $Z=43$ to 73 it is sufficient with $n=7$, giving 23205 evenparity and 179701 odd-parity CSFs. For the highly charged ions we expect relativistic effects, Breit interaction, and QED contributions to be far more important than correlation. For $Z=74$ to 85 it is therefore sufficient with CSF expansions up to $n=6$, resulting in 12541 even-parity and 94265 odd-parity CSFs, and for $Z=86$ to 92 we expand up to $n=5$, which corresponds to a maximum of 5786 even-parity and 41723 odd-parity CSFs.

\section{RESULTS AND DISCUSSION}

\section{A. The magnetic-field-induced $2 s 2 p^{3} P_{0} \rightarrow 2 s^{2}{ }^{1} S_{0}$ E1 transition rates}

Magnetic-field-induced rates of the $2 s^{2}{ }^{1} S_{0}-2 s 2 p^{3} P_{0}$ transition for Be-like ions in a comparatively weak magnetic field can be estimated from the reduced transition rates $A_{\mathrm{MIT}}^{R}$, as defined in Eq. (9). Using this method, we calculate rates for all ions in the beryllium isoelectronic sequence with zero nuclear spin between $Z=5$ and $Z=92$. In these calculations the wave function of the $2 s 2 p^{3} P_{0}$ state under the influence of an external magnetic field is approximately described including $2 s 2 p^{3} P_{1}$ and $2 s 2{ }^{1} P_{1}$ as perturbers. The resulting reduced rates are presented in Table I in the column labeled "full" (since both perturbers are included). It is found that the MIT rates are small and almost constant $\left(\sim 3 \times 10^{-3} \mathrm{~s}^{-1}\right)$ for high- $Z$ ions. These rates will be compared to the expected E1M1 rates in the subsequent section. Note that the reduced MIT rates by definition correspond to an external magnetic-field strength of $1 \mathrm{~T}$.

We also investigate the importance of the $2 s 2{ }^{1} P_{1}$ perturber by comparing our results to a calculation, labeled "no ${ }^{1} P_{1}$ " in Table I, where we only include $2 s 2 p^{3} P_{1}$. The next 
TABLE I. Transition rates of the magnetic-field-induced E1 transition $2 s^{2}{ }^{1} S_{0}-2 s 2 p^{3} P_{0}$. The reduced rates $A_{\mathrm{MIT}}^{R}$ of Eq. (9) are given in two versions, the "full" version, where both perturbers ${ }^{3} P_{1}$ and ${ }^{1} P_{1}$ are included, and the "no ${ }^{1} P_{1}$ " version, where the ${ }^{1} P_{1}$ perturber has been excluded. The difference in percentage of these two approaches is presented in the $\delta$ column. $A_{\mathrm{MIT}}$ are rates for two example field strengths. Note that the square brackets indicate the power of ten.

\begin{tabular}{|c|c|c|c|c|c|c|c|c|c|c|c|c|c|}
\hline \multirow[b]{2}{*}{ Ion } & \multirow[b]{2}{*}{$Z$} & \multicolumn{3}{|c|}{$A_{\mathrm{MIT}}^{R}(Z)\left(\mathrm{s}^{-1} \mathrm{~T}^{-2}\right)$ (reduced) } & \multicolumn{2}{|c|}{$A_{\mathrm{MIT}}(Z, B)\left(\mathrm{s}^{-1}\right)$} & \multirow[b]{2}{*}{ Ion } & \multirow[b]{2}{*}{$Z$} & \multicolumn{3}{|c|}{$A_{\mathrm{MIT}}^{R}\left(\mathrm{~s}^{-1} \mathrm{~T}^{-2}\right)$ (reduced) } & \multicolumn{2}{|c|}{$A_{\mathrm{MIT}}(Z, B)\left(\mathrm{s}^{-1}\right)$} \\
\hline & & Full & No ${ }^{1} P_{1}$ & $\delta_{\%}$ & $0.5 \mathrm{~T}$ & $1.5 \mathrm{~T}$ & & & Full & No ${ }^{1} P_{1}$ & $\delta_{\%}$ & $\mathrm{~T}$ & $\mathrm{~T}$ \\
\hline B & 5 & $078[-2]$ & $4.080[-2]$ & 0.04 & $020[-2]$ & $176[-2]$ & Hit & re & $371[-3]$ & $3.951[-3]$ & 17.21 & $.426[-4]$ & $7.584[-3]$ \\
\hline $\mathrm{C}$ & 6 & $697[-2]$ & $2.701[-2]$ & 0.12 & $.743[-3]$ & $6.069[-2]$ & & 50 & $61[-3]$ & $3.914[-3]$ & & & $7.562[-3]$ \\
\hline $\mathrm{N}$ & 7 & $881[-2]$ & $2.086[-2]$ & 0.24 & $203[-3]$ & $4.683[-2]$ & $\mathrm{Sb}$ & 51 & $356[-3]$ & & & & $7.551[-3]$ \\
\hline $\mathrm{O}$ & 8 & $746[-2]$ & $1.753[-2]$ & 0.43 & $4.364[-3]$ & $3.928[-2]$ & $\mathrm{Te}$ & 52 & $353[-3]$ & $3.856[-3]$ & 15.02 & $.382[-4]$ & $7.544[-3]$ \\
\hline $\mathrm{F}$ & 9 & $500[-2]$ & $1.510[-2]$ & 0.69 & $3.749[-3]$ & $3.374[-2]$ & & 53 & $355[-3]$ & $3.836[-3]$ & & $8.388[-4]$ & $7.549[-3]$ \\
\hline $\mathrm{Ne}$ & 10 & $318[-2]$ & $1.331[-2]$ & & $294[-3]$ & $2.965[-2]$ & $\mathrm{Xe}$ & $T$ & $358[-3]$ & $3.817[-3]$ & & & \\
\hline $\mathrm{Na}$ & 11 & $177[-2]$ & $1.195[-2]$ & 1.48 & $2.943[-3]$ & $2.649[-2]$ & Cs & 55 & $366[-3]$ & $3.805[-3]$ & 13 & $415[-4]$ & $7.573[-3]$ \\
\hline $\mathrm{Mg}$ & 12 & $065[-2]$ & $1.087[-2]$ & 2.02 & $2.663[-3]$ & $2.397[-2]$ & $\mathrm{Ba}$ & 5 & $375[-3]$ & $3.795[-3]$ & 12.44 & $.438[-4]$ & $7.595[-3]$ \\
\hline $\mathrm{Al}$ & 13 & $739[-3]$ & $1.000[-2]$ & 2.68 & $435[-3]$ & & $\mathrm{La}$ & - & $89[-3]$ & & & & \\
\hline $\mathrm{Si}$ & 14 & $0[-3]$ & $9.291[-3]$ & 3.46 & $245[-3]$ & $2.021[-2]$ & $\mathrm{Ce}$ & 58 & $05[-3]$ & $3.791[-3]$ & & $13[-4]$ & $7.662[-3]$ \\
\hline $\mathrm{P}$ & 15 & $0[-3]$ & $8.703[-3]$ & 4.36 & $085[-3]$ & $1.876[-$ & $=0$ & $\mathrm{~J}$ & $25[-3]$ & $5[-3]$ & 10 & -4] & $07[-3]$ \\
\hline$S$ & 16 & $3[-3]$ & $8.212[-3]$ & 5.38 & $948[-3]$ & & & 60 & $48[-3]$ & $2[-3]$ & & -4] & {$[-3]$} \\
\hline $\mathrm{Cl}$ & 17 & $321[-3]$ & $7.798[-3]$ & 6.51 & $30[-3]$ & $1.647[-2]$ & & 61 & $73[-3]$ & $13[-3]$ & & $82[-4]$ & $14[-3]$ \\
\hline $\mathrm{Ar}$ & 18 & $9[-3]$ & 7.44 & 7.7 & $1.727[-3]$ & & & & -3] & 3.8 & & $-4]$ & \\
\hline K & 19 & $7[-3]$ & 7.14 & 9.12 & $-3]$ & & $\mathrm{u}$ & 63 & {$[-3]$} & {$[-3]$} & & $-4]$ & $-3]$ \\
\hline $\mathrm{Ca}$ & 20 & $227[-3]$ & $6.885[-3]$ & 10.56 & $57[-3]$ & 1401[ & & 6 & $64[-3]$ & $3.867[-3]$ & & $11[-4]$ & $8.020[-3]$ \\
\hline $\mathrm{Sc}$ & 21 & & & & & & & & & & & & \\
\hline $\mathrm{Ti}$ & 22 & $9[-3]$ & $6.464[-3]$ & 3 & $1.422[-3]$ & 1.2 & Dy & 6 & $3[-3]$ & $5[-3]$ & & -4] & $-3]$ \\
\hline $\mathrm{V}$ & 23 & $0[-3]$ & 6.29 & 1 & $1.365[-3]$ & 1.22 & & & $4[-3]$ & & & $-4]$ & \\
\hline $\mathrm{Cr}$ & 24 & & & & & & & & 3] & & & & \\
\hline $\mathrm{Mn}$ & 25 & $7[-3]$ & 5.99 & & $57[-3]$ & & $\mathrm{Tm}$ & 69 & $85[-3]$ & $4.039[-3]$ & & & $16[-3]$ \\
\hline $\mathrm{Fe}$ & 26 & $893[-3]$ & {$[-3]$} & 1 & $1.223[-3]$ & 1.10 & & 7 & $38[-3]$ & $4.083[-3]$ & & $4[-4]$ & {$[-3]$} \\
\hline Co & 27 & $3[-3]$ & {$[-3]$} & 3 & $6[-3]$ & 1.0 & $\mathrm{Lu}$ & 71 & $94[-3]$ & $2[-3]$ & & {$[-4]$} & $8.761[-3]$ \\
\hline $\mathrm{Ni}$ & 28 & $2[-3]$ & $5.6 ?$ & & $-3]$ & & & $\pi$ & $51[-3]$ & & & {$[-4]$} & $9[-3]$ \\
\hline $\mathrm{Cu}$ & 29 & $474[-3]$ & $5.523[-3]$ & 2 & $8[-3]$ & 1.00 & & 7 & $10[-3]$ & $4.233[-3]$ & & $02[-3]$ & $9.022[-3]$ \\
\hline $\mathrm{Zn}$ & 30 & $7[-3]$ & $5.418[-3]$ & 24.35 & $1.089[-3]$ & 9.8 & W & 74 & $5[-3]$ & $4.544[-3]$ & & $9[-3]$ & $9.708[-3]$ \\
\hline $\mathrm{Ga}$ & 31 & $49[-3]$ & 5.31 & & $1.062[-3]$ & & & s & $95[-3]$ & & & $99[-3]$ & $9.889[-3]$ \\
\hline $\mathrm{Ge}$ & 32 & $151[-3]$ & $5.214[-3]$ & 25. & $1.038[-3]$ & 9.3 & & 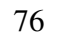 & $477[-3]$ & $4[-3]$ & & $19[-3]$ & $1.007[-2]$ \\
\hline As & 33 & $0[-3]$ & {$[-3]$} & 25.97 & {$[-3]$} & 9.1 & $\mathrm{Ir}$ & 77 & {$[-3]$} & $6[-3]$ & & $-3]$ & $1.027[-2]$ \\
\hline $\mathrm{Se}$ & 34 & $977[-3]$ & $5.017[-3]$ & 26.13 & $9.944[-4]$ & & & 7 & $656[-3]$ & $4.861[-3]$ & & $1.164[-3]$ & $1.048[-2]$ \\
\hline $\mathrm{Br}$ & 35 & $902[-3]$ & $4.921[-3]$ & 26.13 & $9.755[-4]$ & $8.779[-$ & & 7 & $751[-3]$ & $4.951[-3]$ & & $88[-3]$ & $1.069[-2]$ \\
\hline $\mathrm{Kr}$ & 36 & $2[-3]$ & $4.827[-3]$ & 25.97 & $9.581[-4]$ & 8.6 & $\mathrm{Hg}$ & 80 & $6[-3]$ & {$[-3]$} & & $1[-3]$ & $1.090[-2]$ \\
\hline $\mathrm{Rb}$ & 37 & $769[-3]$ & $4.736[-3]$ & 25.67 & $9.422[-4]$ & $8.480[-3]$ & $\mathrm{Tl}$ & 81 & $.945[-3]$ & $5.135[-3]$ & & $1.236[-3]$ & $1.113[-2]$ \\
\hline $\mathrm{Sr}$ & 38 & $.712[-3]$ & $4.649[-3]$ & 25.25 & $9.279[-4]$ & $8.351[-3]$ & $\mathrm{Pb}$ & 02 & $5.046[-3]$ & $5.231[-3]$ & 3.67 & $1.262[-3]$ & $1.135[-2]$ \\
\hline $\mathrm{Y}$ & 39 & $.660[-3]$ & $4.564[-3]$ & 24.72 & $9.149[-4]$ & $8.234[-3]$ & $\mathrm{Bi}$ & 83 & $5.151[-3]$ & $5.332[-3]$ & & $1.288[-3]$ & $1.159[-2]$ \\
\hline $\mathrm{Zr}$ & 40 & $614[-3]$ & $4.485[-3]$ & 24.11 & $9.034[-4]$ & $8.131[-3]$ & Po & 8 & $5.261[-3]$ & $5.437[-3]$ & 3.35 & $1.315[-3]$ & $1.184[-2]$ \\
\hline $\mathrm{Nb}$ & 41 & $.572[-3]$ & & & $8.930[-4]$ & & 10 & 85 & & $5.542[-$ & & & \\
\hline Mo & 42 & $3.533[-3]$ & $4.335[-3]$ & 22.71 & $8.833[-4]$ & $7.949[-3]$ & $\mathrm{Rn}$ & 86 & $5.451[-3]$ & $5.617[-3]$ & 3.06 & $1.363[-3]$ & $1.226[-2]$ \\
\hline $\mathrm{Tc}$ & 43 & $495[-3]$ & $4.263[-3]$ & 21.96 & $8.738[-4]$ & & $\mathrm{Fr}$ & 8 & $5.555[-3]$ & $5.718[-3]$ & 2.92 & $1.389[-3]$ & $1.250[-2]$ \\
\hline $\mathrm{Ru}$ & 44 & $3.466[-3]$ & $4.200[-3]$ & 21.17 & $8.665[-4]$ & $7.799[-3]$ & $\mathrm{Ra}$ & 88 & $5.641[-3]$ & $5.799[-3]$ & 2.79 & $1.410[-3]$ & $1.269[-2]$ \\
\hline $\mathrm{Rh}$ & 45 & $3.441[-3]$ & $4.142[-3]$ & 20.37 & $8.602[-4]$ & $7.742[-3]$ & Ac & 89 & $5.737[-3]$ & $5.890[-3]$ & 2.66 & $1.434[-3]$ & $1.291[-2]$ \\
\hline $\mathrm{Pd}$ & 46 & $3.419[-3]$ & $4.088[-3]$ & 19.57 & $8.548[-4]$ & $7.693[-3]$ & Th & 90 & $5.816[-3]$ & $5.964[-3]$ & 2.54 & $1.454[-3]$ & $1.309[-2]$ \\
\hline $\mathrm{Ag}$ & 47 & $3.401[-3]$ & $4.040[-3]$ & 18.77 & $8.503[-4]$ & $7.653[-3]$ & $\mathrm{Pa}$ & 91 & $5.914[-3]$ & $6.057[-3]$ & 2.43 & $1.478[-3]$ & $1.331[-2]$ \\
\hline $\mathrm{Cd}$ & 48 & $3.385[-3]$ & $3.994[-3]$ & 17.99 & $8.464[-4]$ & $7.617[-3]$ & $\mathrm{U}$ & 92 & $5.978[-3]$ & $6.116[-3]$ & 2.32 & $1.494[-3]$ & $1.345[-2]$ \\
\hline
\end{tabular}

column in Table I, labeled " $\delta \%$," shows the difference between these two approaches as a percentage. It is clear that excluding ${ }^{1} P_{1}$ leads to a significant overestimation of the rates, by more than $5 \%$ for $Z=16$ to 75 , reaching as much as $26 \%$ for $\mathrm{Be}-$ like $\mathrm{Se}$ and $\mathrm{Br}$. The full calculation reduces the MIT rates, compared to only including ${ }^{1} P_{1}$, due to the cancellation of the individual transition amplitudes from the two perturbers involved in Eq. (8).
The two remaining columns of Table I (labeled " $B$ ") show two example field strengths of 0.5 and $1.5 \mathrm{~T}$, respectively, where the latter is the maximum field strength of the dipole bending magnets of ESR, as mentioned in Sec. II. Note that the bending magnets only cover parts of the storage ring, resulting in a smaller effective field. Details of results connected to the particular experiment suggested at ESR are presented in Sec. V D. 
TABLE II. Comparison of excitation energies $E$, energy separations with the ${ }^{3} P_{0}$ reference state $\Delta E$, and transition rates $A$ involved in the MIT calculation for some selected charge states. We compare them with experimental energies [46] and theoretical rates [48] from the NIST database [47] for $Z=5$. Results for ions of charge states $Z=50$ and 92 are compared with another recent accurate theoretical work [17] (labeled BSRCI). The fractional difference relative to our results is given in rows labeled $\delta \%$. All energies are given in units of $\mathrm{cm}^{-1}$, transition rates are in $\mathrm{s}^{-1}$, and numbers in brackets represent powers of ten. Note that our values are rounded off to the same number of digits as given in the data to which they are compared.

\begin{tabular}{|c|c|c|c|c|c|c|c|c|}
\hline Ion & Source & $E_{{ }^{3} P_{0}}$ & $\Delta E_{{ }^{3} P_{0}-{ }^{3} P_{1}}$ & $E_{{ }^{3} P_{1}}$ & $\Delta E_{{ }^{3} P_{0}-{ }^{1} P_{1}}$ & $E_{{ }^{P_{P_{1}}}}$ & $A^{{ }^{3} P_{1} \rightarrow{ }^{1} S_{0}}$ & $A^{{ }^{1} P_{1} \rightarrow{ }^{1} S_{0}}$ \\
\hline \multirow[t]{3}{*}{$\mathrm{B}_{Z=5}$} & this work & $3.727186[4]$ & $6.06[0]$ & $3.727792[4]$ & $3.62225[4]$ & $7.349438[4]$ & $1.03[1]$ & $1.20[9]$ \\
\hline & NIST & $3.733554[4]$ & $6.11[0]$ & $3.734165[4]$ & $3.60610[4]$ & $7.339651[4]$ & $1.04[1]$ & $1.20[9]$ \\
\hline & $\delta_{\%}$ & 0.17 & 0.82 & 0.17 & -0.45 & -0.13 & 0.97 & 0.00 \\
\hline \multirow[t]{3}{*}{$\mathrm{Sn}_{Z=50}$} & this work & $7.58328[5]$ & $1.63389[5]$ & $9.21716[5]$ & $2.5829[6]$ & $3.34121[6]$ & $1.652[9]$ & $3.819[11]$ \\
\hline & BSRCI & $7.58449[5]$ & $1.63306[5]$ & $9.21755[5]$ & $2.5807[6]$ & $3.33919[6]$ & $1.676[9]$ & $3.825[11]$ \\
\hline & $\delta_{\%}$ & 0.02 & -0.05 & 0.00 & -0.09 & -0.06 & 1.45 & 0.16 \\
\hline \multirow[t]{3}{*}{$\mathrm{U}_{Z=92}$} & this work & $2.10309[6]$ & $3.2251[5]$ & $2.42561[6]$ & $3.4277[7]$ & $3.63804[7]$ & $9.806[9]$ & $1.169[14]$ \\
\hline & BSRCI & $2.07739[6]$ & $3.2241[5]$ & $2.39980[6]$ & $3.4230[7]$ & $3.63083[7]$ & $9.773[9]$ & $1.166[14]$ \\
\hline & $\delta_{\%}$ & -1.22 & -0.03 & -1.06 & -0.14 & -0.20 & 0.34 & -0.26 \\
\hline
\end{tabular}

\section{B. Uncertainties of results}

In order to benchmark the quality of our wave functions (and ultimately the magnetic-field-induced transition rates) we compare our obtained excitation energies, energy separations between the reference state ${ }^{3} P_{0}$ and the perturbers ${ }^{1,3} P_{1}$, and the involved $J$-dependent transition rates with experiment [46] and another accurate theoretical work based on B-spline relativistic configuration interaction calculations [17] with a careful treatment of QED effects (from here on this calculation will be referred to as BSRCI for brevity). In this section we also present results from convergence studies of the involved parameters and the influence of QED effects, and finally, we investigate the impact of neglecting additional perturbers.

This initial comparison with experiment and other theory is presented in Table II for three selected charge states representing the neutral end and the intermediate and the highly charged ions, which allows us to test our model with respect to correlation and relativistic and QED effects. Note that the excitation energies of ${ }^{3} P_{1}$ and ${ }^{1} P_{1}$ are, different from the other parameters presented, of no direct importance in the evaluation of the MIT rates. On the other hand, the accuracy of, e.g., the energy separations between the reference state and the perturbers is of particular interest as it enters directly in the evaluation of the magnetic mixing coefficients $d_{\Gamma J}$ in Eq. (3). The same goes for the E1 transition matrix elements of ${ }^{3} P_{1} \rightarrow{ }^{1} S_{0}$ and ${ }^{1} P_{1} \rightarrow{ }^{1} S_{0}$.

The first ion, chosen to represent the neutral end in this test, is singly ionized boron $(Z=5)$. We expect energies of ions this close to neutral to be dominated by contributions from electron correlation (relative to Dirac-Fock energies). It can be seen from Table II that both excitation and separation energies are in excellent agreement with experiment, in most cases well within $1 \%$. Tin $(Z=50)$ is chosen to represent the intermediate part of the sequence. This should be a simple calculation in our approach as both correlation and QED effects are small and other relativistic effects are taken care of efficiently, which is apparent from the resulting energies as the difference from BSRCI is almost negligible. For the high end of the sequence we choose the ion of the highest charge state in this work, namely, uranium $(Z=92)$. There is a slightly larger disagreement between our excitation energies and the BSRCI results for the intermediate ions (as expected due to a greater influence from QED effects), with a maximum deviation of $1.22 \%$. The energy separations, however, are in very good agreement with the BSRCI energies.

We continue with the convergence of the MIT rates and the involved parameters as the active set of orbitals is increased according to the model presented in Sec. IV. We conclude from our studies that the MIT rates are converged to $5 \%$ on the far neutral end of the sequence, whereas the rates for the highly charged ions are almost fully converged, as expected.

The QED contribution to the MIT rate ranges from zero in the neutral end to $-15 \%$ for $Z=50$ and about $-40 \%$ for $Z=92$. In our relatively simple QED model, we estimate an upper limit of the QED uncertainty for the mid- $Z$ ions, to about 5\%. As the impact of QED is almost half of the total rate for the highly charged ions, the errors are most certainly larger as well. This is, however, of no direct problem to this work as the E1M1 rate dominates over the MIT rate for these ions by more than two orders of magnitude for $Z=75$ and higher (see Fig. 3). Note, however, that the formula for the E1M1 rate by Laughlin, Eq. (13) [23], which is used in this work for reference values, is nonrelativistic and ignores the ${ }^{1} P_{1}$ state and should therefore be used with care, especially for the highly charged ions. The competition between the MIT and E1M1 rates will be discussed further in Sec. V C.

Estimating the influence of including perturbers other than $2 s 2 p^{1} P_{1}$ and $2 s 2 p^{3} P_{1}$ still remains. Starting with $Z=5$, we evaluate the size of the magnetic-field-induced mixing, Eq. (3), with the closest-lying energy level of odd parity above the $2 s 2 p^{1} P_{1}$ level. This is state $2 s 3 p^{3} P_{1}$, having an energy separation from the $2 s 2 p^{3} P_{0}$ reference state of $106655 \mathrm{~cm}^{-1}$ according to the NIST ASD [47]. Furthermore the matrix element involved in the evaluation of the mixing coefficient is about a factor of 10 smaller for $2 s 3 p^{3} P_{1}$, which implies a mixing coefficient a total of 30 times smaller than the one with 2s $2 p^{1} P_{1}$. The same line of action for $Z=50$ gives a mixing coefficient with $2 s 3 p^{3} P_{1}$ that is more than 1500 times smaller than the one for $2 s 2 p^{1} P_{1}$. Calculating the MIT rates including the $2 s 3 p$ perturbers reveals an additional contribution of about $0.01 \%$ for $Z=92$, which reduces to zero for $Z=5$, and we 


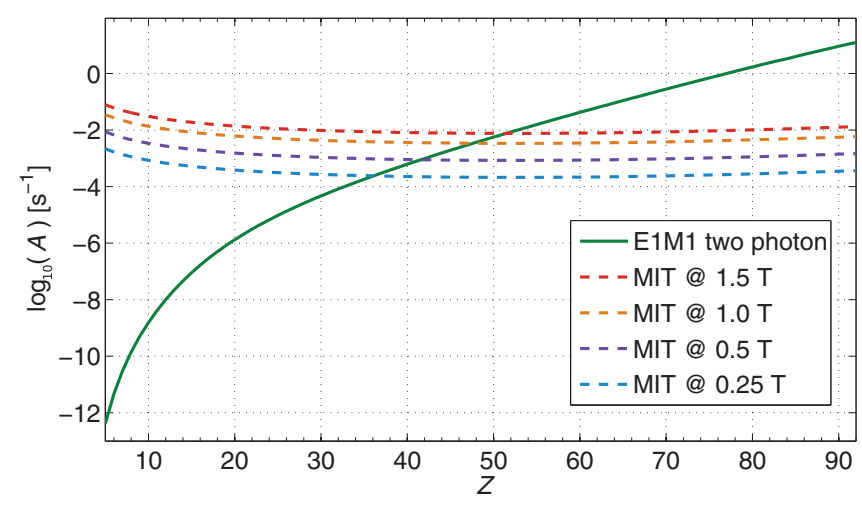

FIG. 3. (Color online) Comparison of transition rates for the two different decay channels of the $2 s 2 p^{3} P_{0} \rightarrow 2 s^{2}{ }^{1} S_{0}$ transition in Be-like atoms with zero nuclear spin under the influence of an external magnetic field. The dashed lines shows how the magneticfield-induced transition (MIT) rate varies along the isoelectronic sequence, and the green solid line shows the behavior of the E1M1 two-photon transition rate as predicted by Eq. (13). The rates are given in $\log _{10}$ scale.

conclude that neglecting further perturbers has a negligible impact on the MIT rates.

We end the discussion of this section by giving an estimation of the total MIT transition-rate uncertainty. After a careful convergence study we concluded that the rates were converged to within $5 \%$ to $0 \%$ from low- to high- $Z$ ions. We estimate the accuracy of the QED contribution for the low- to mid- $Z$ ions to be $0 \%-5 \%$ and around $10 \%$ for the highly charged ions. The influence of neglecting further perturbers is very small, and thus we estimate the total accuracy of our MIT rates to vary from about $5 \%$ for low- and mid- $Z$ ions up to $10 \%$ as an upper limit for the highly charged ions, where it should be clear that the errors related to QED are very hard to evaluate.

\section{The competition between the E1M1 two-photon and MIT decays}

To be able to extract the E1M1 two-photon transition rate, one needs to determine, or at least estimate, the MIT rate, which was done in Sec. V A. In order to predict the influence of the magnetic field on the total lifetime, one should compare these MIT rates to the corresponding two-photon transition rates. We begin, however, by evaluating the MIT rates further, along the sequence.

According to the scaling law for physical quantities in the hydrogenic approximation, the MIT rate is roughly proportional to $Z^{4}$ for high- $Z$ ions. To obtain a better description of the dependence of the MIT rate on the atomic number, we perform a nonlinear least-squares fit of the calculated reduced rates including an extra general term of $Z$, resulting in the following expression:

$$
A_{\mathrm{MIT}}^{R}(Z)=\alpha Z^{\delta}+\beta Z^{4}+\gamma,
$$

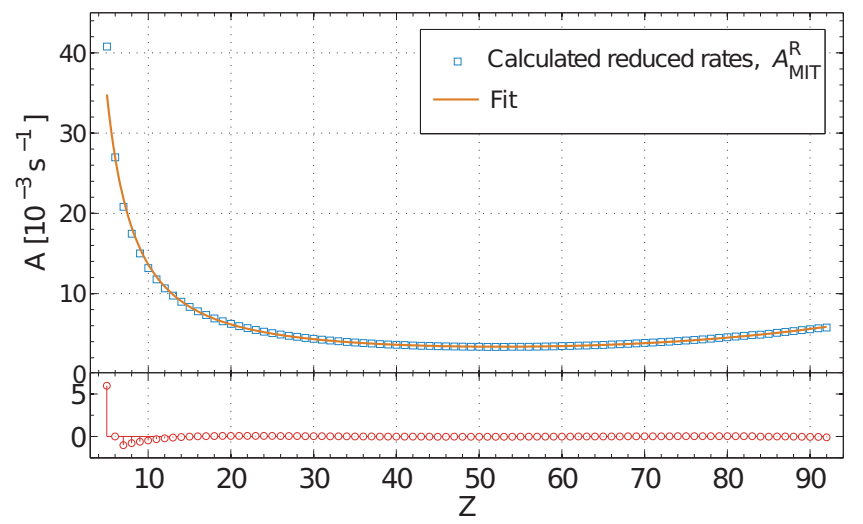

FIG. 4. (Color online) Reduced transition rates of the magneticfield-induced transition $2 s 2 p^{3} P_{0} \rightarrow 2 s^{2}{ }^{1} S_{0}$ due to mixing with the intercombination channel (see Fig. 2), which in turn is allowed due to mixing with the ${ }^{1} P_{1}$ level. The solid line shows the least-squares fit presented in Eq. (10). The residuals of the fit are shown in the bottom panel.

where

$$
\begin{array}{ll}
\alpha=3.703 \times 10^{-1} \mathrm{~s}^{-1} \mathrm{~T}^{-2}, & \beta=4.717 \times 10^{-11} \mathrm{~s}^{-1} \mathrm{~T}^{-2}, \\
\gamma=2.074 \times 10^{-3} \mathrm{~s}^{-1} \mathrm{~T}^{-2}, & \delta=-1.507 .
\end{array}
$$

The first term in Eq. (10) dominates at the neutral and intermediate parts of the sequence and accounts for the deviation from the hydrogenic behavior and corresponds to, e.g., stronger correlation effects. The $Z^{4}$ dependence increasingly makes up the major part of the rate for about $Z=70$ and higher, as expected from the hydrogenic scaling laws. A plot of the computed MIT rate data points of Table I and the fitted curve are presented in Fig. 4 together with the deviation of the fit in the panel below the main plot. As can be seen from Fig. 4, the fit is in excellent agreement with the calculations, except for a few ions at the neutral end of the sequence, where the reduced transition rates of Table I should be used instead if there is a need for high accuracy.

Using Eq. (9), we can write down a final formula for the nonreduced MIT rate,

$$
A_{\mathrm{MIT}}(Z, B)=A_{\mathrm{MIT}}^{R}(Z) B^{2}=\left[\alpha Z^{\delta}+\beta Z^{4}+\gamma\right] B^{2},
$$

with the constants $\alpha, \beta, \gamma$, and $\delta$ given by Eq. (11).

For the E1M1 decay from $2 s 2 p^{3} P_{0}$ in Be-like ions only two theoretical predictions, those by Schmieder [49] and Laughlin [23], are available. As mentioned in the previous section, Laughlin's derivation is nonrelativistic and ignores the $2 s 2 p^{1} P_{1}$ state. Thus all comparisons between the MIT and E1M1 transition rates in this section should be read with the knowledge that the accuracy of the E1M1 rates could be very low for high- $Z$ ions. Bernhardt et al. [25] evaluated the integrals involved in Laughlins's expression for the rate analytically, with the resulting formula

$$
\begin{aligned}
A_{\mathrm{E} 1 \mathrm{M} 1}(E, \Delta, Z)= & \frac{1}{6} A_{0} Z^{4}\left[E^{5}-8 E^{4} \Delta-68 E^{3} \Delta^{2}-120 E^{2} \Delta^{3}-60 E \Delta^{4}\right. \\
& \left.+\frac{12 \Delta^{2}\left(3 E^{2}+10 E \Delta+10 \Delta^{2}\right)(E+\Delta)^{2}}{E+2 \Delta} \ln \left(\frac{E+\Delta}{\Delta}\right)\right],
\end{aligned}
$$




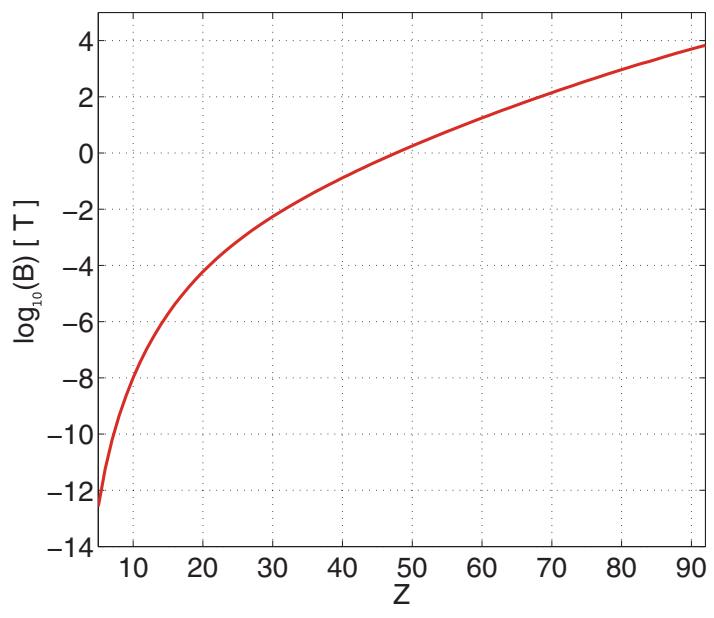

FIG. 5. (Color online) The critical magnetic-field strength $B^{\text {critical }}$, as defined in Eq. (14), along the Be-like sequence in $\log _{10}$ scale. These field strengths correspond an MIT rate $A_{\mathrm{MIT}}$ equal to the E1M1 two-photon rate $A_{\mathrm{E} 1 \mathrm{M} 1}$.

where $E$ represents the excitation energy of the $2 s 2 p^{3} P_{0}$ state, $\Delta$ is the $2 s 2 p^{3} P_{0,1}$ fine-structure splitting, and $A_{0}=2.867 \times$ $10^{-11} \mathrm{~s}^{-1}$.

In Fig. 3 we illustrate the E1M1 two-photon transition rates along the Be-like sequence according to this formula. In Fig. 3 we also include the MIT rates calculated with some examples of magnetic-field strengths between $B=0.25$ and 1.5 T. From Fig. 3 it can be seen that the rates of the two transition channels are, in general, of comparable size for mid- $Z$ ions. At the low$Z$ end of the sequence the MIT becomes the dominating decay channel, while for high- $Z$ ions the E1M1 channel has a much faster rate. Furthermore we define a critical magnetic-field strength $B^{\text {critical }}$ as the field corresponding to a MIT rate equal to the E1M1 rate, leading to the following relation:

$$
B^{\text {critical }} \equiv \sqrt{\frac{A_{\mathrm{E} 1 \mathrm{M} 1}}{A_{\mathrm{MIT}}^{R}}} .
$$

Using Eq. (13) for the E1M1 rates (keeping well aware of the possible low accuracy of the E1M1 transition rates for the highly charged ions as pointed out earlier) and our reduced MIT rates from Table I, we calculate the critical magnetic-field strength along the sequence (see Fig. 5). From Fig. 5 one can readily estimate in which region of $Z$ and $B$ the impact of the magnetic field on the lifetime of ${ }^{3} P_{0}$ is of significant importance. For example, for $\mathrm{Sn}, Z=50$, the critical magnetic field is about $0.3 \mathrm{~T}$. Hence it is crucial to include and evaluate the MIT rate in measurements involving an external magnetic field of this magnitude.

\section{The E1M1 transition-rate measurement at ESR}

Considering the particular case of the proposed experiment at ESR, the six dipole bending magnets cover $36.24 \%$ of the ring, as explained in Sec. II. Each one of these magnets has a constant and near-homogeneous magnetic field that can be set to any field strength $B_{\text {magnets }}^{\text {bending }}$ up to a maximum of $1.5 \mathrm{~T}$, depending on the mass and energy of the stored ion. Hence we may conclude that, using Eq. (12), the effective rate of the $2 s 2 p^{3} P_{0} \rightarrow 2 s^{2} S_{0}$ transition in a measurement at ESR can be estimated from

$$
\begin{aligned}
A_{\mathrm{eff}}^{\mathrm{ESR}}\left(Z, B_{\mathrm{eff}}\right) & =A_{\mathrm{MII}}^{\mathrm{ESR}}\left(Z, B_{\mathrm{eff}}\right)+A_{\mathrm{E} 1 \mathrm{M} 1}(Z) \\
& =B_{\mathrm{eff}}^{2} A_{\mathrm{MIT}}^{R}(Z)+A_{\mathrm{E} 1 \mathrm{M} 1}(Z) \\
& =B_{\mathrm{eff}}^{2}\left[\alpha Z^{\delta}+\beta Z^{4}+\gamma\right]+A_{\mathrm{E} 1 \mathrm{M} 1}(Z),
\end{aligned}
$$

where $B_{\text {eff }}=\sqrt{0.3624} B_{\text {magnets }}^{\text {bending }}$ is the effective magnetic-field strength deduced from averaging the MIT rate over one full revolution of ESR. The effective field strength is variable up to a maximum of $0.90 \mathrm{~T}$.

One can then choose to use either the reduced transition rates $A_{\mathrm{MIT}}^{R}$, given in Table I, or simply the $Z$-dependent function in square brackets in Eq. (15) with fitted parameters defined in Eq. (11). The measured total lifetime of ${ }^{3} P_{0}$ can then be directly related to this rate, from which an approximation of the E1M1 transition rate can be obtained.

\section{CONCLUSIONS}

In summary, a proposal [24-26] has been made to measure the rate of the $2 s 2 p^{3} P_{0} \rightarrow 2 s^{2} S_{0} \quad$ E1M1 two-photon transition in heavy Be-like ions with zero nuclear spin at the heavy-ion storage-ring ESR of the GSI Helmholtz Center for Heavy-Ion Research in Darmstadt, Germany. The E1M1 two-photon transition is the lowest-order transition for Be-like isotopes with zero nuclear spin in a field-free environment. In a storage ring, however, the bending magnets generate magnetic fields, which possibly could have a large impact on the lifetime of the ${ }^{3} P_{0}$ level through magnetic-field quenching. In this work we therefore present accurate theoretical transition rates of the magnetic-field-induced transition $2 s 2 p^{3} P_{0} \rightarrow 2 s^{2}{ }^{1} S_{0}$ in Be-like ions with the purpose of aiding such storage-ring measurements.

Our theoretical approach is based on accurate wave functions calculated in an MCDHF scheme followed by a large-scale RCI calculation, as described in Secs. III and IV. The MIT rates can then be obtained through Eq. (8), and we presented results and discussion in Sec. V. The transition rates are presented in Table I and also as a function of the charge state $Z$ of the ion of interest and the magnetic-field strength $B$ [see Eq. (12)]. The quality of our results is motivated through a comparison with experiments and other theoretical results as well as convergence studies, the impact of QED effects, and an evaluation of the influence of neglecting further perturbers. We conclude that our errors are within 5\% for low and mid $Z$ and slightly higher for the highly charged ions due to larger QED effects.

We continued by investigating how big an impact the external $B$ field would have on the total measured lifetime of the ${ }^{3} P_{0}$ level compared to the lifetime associated with the E1M1 two-photon transition. In Fig. 3 an approximate theoretical prediction of the E1M1 transition rate, Eq. (13), is compared to our calculated MIT rates for some different typical field strengths. Figure 3 shows that storage-ring measurements in general, involving external magnetic fields, are infeasible for ions at the neutral end of the isoelectronic sequence where the MIT channel completely dominates. However, foremost, we can conclude that in order to determine the E1M1 rate from such measurements, an accurate evaluation of the MIT rates is crucial for ions around $Z=25$ to 65 , where the MIT and E1M1 transition rates are of the same order of magnitude. 
Finally, we applied our results to the particular measurement proposed at ESR. We introduced an effective magnetic field $B_{\text {eff }}$, depending on the bending magnet setup of ESR, and gave a relation, Eq. (15), for the total transition rate corresponding to the measured lifetime of ${ }^{3} P_{0}$. From this expression one can then, if the total transition rate can be evaluated experimentally, readily obtain an estimation of the rate associated with the E1M1 two-photon transition.

\section{ACKNOWLEDGMENTS}

J.G., J.L., T.B., and P.J. gratefully acknowledge support from the Swedish Research Council (Vetenskapsrådet) and the Swedish Institute under the Visby program. D.B. and S.S. were supported by Deutsche Forschungsgeinschaft (DFG) under Contract No. Schi378/8-1.
[1] L. Spitzer, Jr. and J. L. Greenstein, Astrophys. J. 114, 407 (1951).

[2] M. A. Dopita, L. Binette, and R. D. Schwartz, Astrophys. J. 261, 183 (1982).

[3] D. E. Osterbrock and G. J. Ferland, Astrophysics of Gaseous Nebulae and Active Galactic Nuclei, 2nd ed. (University Science Books, Sausalito, CA, 2006).

[4] M. Göppert-Mayer, Ann. Phys. (Berlin, Gerrmany) 401, 273 (1931).

[5] P. H. Mokler and R. W. Dunford, Phys. Scr. 69, C1 (2004).

[6] P. Amaro, J. P. Santos, F. Parente, A. Surzhykov, and P. Indelicato, Phys. Rev. A 79, 062504 (2009).

[7] A. Surzhykov, J. P. Santos, P. Amaro, and P. Indelicato, Phys. Rev. A 80, 052511 (2009).

[8] B. J. Wundt and U. D. Jentschura, Phys. Rev. A 80, 022505 (2009).

[9] D. Solovyev, V. Dubrovich, A. V. Volotka, L. Labzowsky, and G. Plunien, J. Phys. B 43, 175001 (2010).

[10] S. Trotsenko et al., Phys. Rev. Lett. 104, 033001 (2010).

[11] A. Surzhykov, A. Volotka, F. Fratini, J. P. Santos, P. Indelicato, G. Plunien, T. Stöhlker, and S. Fritzsche, Phys. Rev. A 81, 042510 (2010).

[12] F. Fratini, M. C. Tichy, T. Jahrsetz, A. Buchleitner, S. Fritzsche, and A. Surzhykov, Phys. Rev. A 83, 032506 (2011).

[13] I. M. Savukov and W. R. Johnson, Phys. Rev. A 66, 062507 (2002).

[14] L. N. Labzowsky and A. V. Shonin, Phys. Rev. A 69, 012503 (2004).

[15] T. Brage, P. G. Judge, A. Aboussaïd, M. R. Godefroid, P. Jönsson, A. Ynnerman, C. F. Fischer, and D. S. Leckrone, Astrophys. J. 500, 507 (1998).

[16] J. P. Marques, F. Parente, and P. Indelicato, Phys. Rev. A 47, 929 (1993).

[17] K. T. Cheng, M. H. Chen, and W. R. Johnson, Phys. Rev. A 77, 052504 (2008).

[18] M. Andersson, Y. Zou, R. Hutton, and T. Brage, Phys. Rev. A 79, 032501 (2009).

[19] J. G. Li and C. Dong, Plasma Sci. Technol. (Bristol, UK) 12, 364 (2010).

[20] J. Li, C. Dong, P. Jönsson, and G. Gaigalas, Phys. Lett. A 375, 914 (2011).

[21] S. Schippers, E. W. Schmidt, D. Bernhardt, D. Yu, A. Müller, M. Lestinsky, D. A. Orlov, M. Grieser, R. Repnow, and A. Wolf, Phys. Rev. Lett. 98, 033001 (2007).

[22] S. Schippers, D. Bernhardt, A. Müller, M. Lestinsky, M. Hahn, O. Novotný, D. W. Savin, M. Grieser, C. Krantz, R. Repnow, and A. Wolf, Phys. Rev. A 85, 012513 (2012).

[23] C. Laughlin, Phys. Lett. A 75, 199 (1980).

[24] S. Schippers, D. Bernhardt, A. Müller, C. Brandau, S. Fritzsche, C. Kozhuharov, M. Lestinsky, T. Stöhlker, and Z. Harman,
GSI Helmholtzzentrum für Schwerionenforschung, Darmstadt, Germany, Proposal No. E118, 2011 (unpublished).

[25] D. Bernhardt, C. Brandau, C. Kozhuharov, A. Müller, S. Schippers, S. Böhm, F. Bosch, J. Jacobi, S. Kieslich, H. Knopp, P. H. Mokler, F. Nolden, W. Shi, Z. Stachura, M. Steck, and T. Stöhlker, J. Phys. Conf. Ser. 388, 012007 (2012).

[26] S. Schippers, in Eighth International Conference on Atomic and Molecular Data and Their Applications: ICAMDATA-2012, AIP Conf. Proc. No. 1545 (AIP, New York, 2013), p. 7.

[27] P. Beiersdorfer, J. H. Scofield, and A. L. Osterheld, Phys. Rev. Lett. 90, 235003 (2003).

[28] E. Träbert, J. Phys. B: At. Mol. Opt. Phys. 43, 074034 (2010).

[29] M. Grieser et al., Eur. Phys. J. Spec. Top. 207, 1 (2012).

[30] H. Poth, Phys. Rep. 196, 135 (1990).

[31] F. Caspers and D. Möhl, Eur. Phys. J. H 36, 601 (2012).

[32] S. Mannervik, J. Lidberg, L. O. Norlin, and P. Royen, Phys. Rev. A 56, R1075 (1997).

[33] S. Mannervik, J. Lidberg, L.-O. Norlin, P. Royen, A. Schmitt, W. Shi, and X. Tordoir, Phys. Rev. Lett. 83, 698 (1999).

[34] E. Träbert, M. Grieser, J. Hoffmann, C. Krantz, S. Reinhardt, R. Repnow, A. Wolf, and P. Indelicato, New J. Phys. 13, 023017 (2011).

[35] H. T. Schmidt, P. Forck, M. Grieser, D. Habs, J. Kenntner, G. Miersch, R. Repnow, U. Schramm, T. Schüssler, D. Schwalm, and A. Wolf, Phys. Rev. Lett. 72, 1616 (1994).

[36] B. Franzke, Nucl. Instrum. Methods Phys. Res., Sect. B 24-25, 18 (1987).

[37] M. Maul, A. Schäfer, and P. Indelicato, J. Phys. B 31, 2725 (1998)

[38] J. Li, J. Grumer, W. Li, M. Andersson, T. Brage, R. Hutton, P. Jönsson, Y. Yang, and Y. Zou, Phys. Rev. A 88, 013416 (2013).

[39] K. T. Cheng and W. J. Childs, Phys. Rev. A 31, 2775 (1985).

[40] I. P. Grant, Relativistic Quantum Theory of Atoms and Molecules (Springer, New York, 2007).

[41] P. Jönsson, G. Gaigalas, J. Bieroń, C. F. Fischer, and I. Grant, Comput. Phys. Commun. 184, 2197 (2013).

[42] M. Andersson and P. Jönsson, Comput. Phys. Commun. 178, 156 (2008).

[43] K. Dyall, I. Grant, C. Johnson, F. Parpia, and E. Plummer, Comput. Phys. Commun. 55, 425 (1989).

[44] J. Olsen, M. R. Godefroid, P. Jönsson, P. A. Malmqvist, and C. F. Fischer, Phys. Rev. E 52, 4499 (1995).

[45] P. Jönsson and C. Froese Fischer, Phys. Rev. A 57, 4967 (1998).

[46] A. N. Ryabtsev, I. Kink, Y. Awaya, J. O. Ekberg, S. Mannervik, A. Ölme, and I. Martinson, Phys. Scr. 71, 489 (2005).

[47] A. Kramida, Y. Ralchenko, J. Reader, and NIST ASD Team, NIST Atomic Spectra Database (version 5.0), http://www.nist.gov/pml/data/asd.cfm.

[48] G. Tachiev and C. F. Fischer, J. Phys. B 32, 5805 (1999).

[49] R. W. Schmieder, Phys. Rev. A 7, 1458 (1973). 